\title{
The Success of Natural Products in Drug Discovery
}

\section{Mouhssen Lahlou}

Service des Sciences de la Vie, Ministère de l’Enseignement Supérieur, de la Recherche Scientifique et de la Formation des Cadres, Rabat, Morocco.

Email: lahloumoh@hotmail.com

Received April 25 ${ }^{\text {th }}, 2013$; revised May 27, 2013 ; accepted June $5^{\text {th }}, 2013$

Copyright (c) 2013 Mouhssen Lahlou. This is an open access article distributed under the Creative Commons Attribution License, which permits unrestricted use, distribution, and reproduction in any medium, provided the original work is properly cited.

\begin{abstract}
Drug discovery leading to robust and viable lead candidates' remains a challenging scientific task, which is the transition from a screening hit to a drug candidate, requires expertise and experience. Natural products and their derivatives have been recognized for many years as a source of therapeutic agents and of structural diversity. However, in addition to their chemical structure diversity and their biodiversity, the development of new technologies has revolutionized the screening of natural products in discovering new drugs. Applying these technologies compensates for the inherent limitations of natural products and offers a unique opportunity to re-establish natural products as a major source for drug discovery. The present article attempts to describe the utilization of compounds derived from natural resources as drug candidates, with a focus on the success of these resources in the process of finding and discovering new and effective drug compounds, an approach commonly referred to as "natural product drug discovery".
\end{abstract}

Keywords: Biodiversity; Drug Discovery; Efficacy; Natural Products; New Technologies; Structural and Chemical Diversity; Success Steps

\section{Introduction}

Natural products, including plants, animals and minerals have been the basis of treatment of human diseases [1,2]. Nevertheless, ancient wisdom has been the basis of modern medicine and will remain as one important source of future medicine and therapeutics.

History of medicine dates back practically to the existence of human civilization. Historically, the majority of new drugs have been generated from natural products (secondary metabolites) and from compounds derived from natural products [1].

Before 20th century, crude and semi-pure extracts of plants, animals, microbes and minerals represented the only medications available to treat human and domestic animal illnesses. The 20th century revolutionized the thinking in the use of drugs, as the receptor theory of drug action. The idea that effect of drug in human body are mediated by specific interactions of the drug molecule with biological macromolecules (proteins or nucleic acids in most cases) led scientist to the conclusion that individual chemical compounds in extracts, rather than some mystical "power of life" are the factors required for the biological activity of the drug. This lead to the beginning of a totally new era in pharmacology, as pure, iso- lated chemicals, instead of extracts, became the standard treatments for diseases. Indeed, many bioactive compounds, responsible for the effects of crude extract drugs, and their chemical structure was elucidated.

Classical examples of drug compounds discovered this way are morphine, the active agent in Opium, and digoxin, a heart stimulant originating from flower Digitalis lanata. The evolution in synthetic chemistry also led to chemical synthesis of many of the elucidated structures.

On the other hand, the R\&D thrust in the pharmaceutical sector is focused on development of new drugs, innovative/indigenous processes for known drugs and development of plant-based drugs through investigation of leads from the traditional systems of medicine [2]. The exploitation of structural chemical databases consisting of a wide variety of chemotypes, in conjunction with databases on target genes and proteins, will surely facilitate the creation of new chemical entities through computational molecular modeling for pharmacological evaluation [3].

Recently, there has been a renewed interest in natural product research due to the failure of alternative drug discovery methods to deliver many lead compounds in key therapeutic areas such as immunosuppression, anti- 
infectives, and metabolic diseases.

Natural products research continues to explore a variety of lead structures, which may be used as templates for the development of new drugs by the pharmaceutical industry. There is no doubt that natural products have been, and will be, important sources of new pharmaceutical compounds [1].

Although traditionally natural products have played an important role in drug discovery, in the past few years most Big Pharma companies have either terminated or considerably scaled down their natural product operations. This is despite a significant number of natural product-derived drugs being ranked worldwide selling ethical drugs in the last years. The process in natural product drug discovery usually required several separation circles and structure elucidation and was thus timeconsuming.

New approaches to improve and accelerate the joint drug discovery and development process are expected to take place mainly from innovation in drug target elucidation and lead structure discovery. Powerful new technologies such as automated separation techniques, highthroughput screening and combinatorial chemistry are revolutionizing drug discovery [2].

The present paper discusses and examines how natural products have produced successful results for the pharmaceutical industry for drug discovery and development, and why nature still remains as important source of new drug compounds until today. It's also offers a broad overview of the newest techniques being used in pharmaceutical research today for drug discovery.

\section{Nature as Source of New Drug Compounds}

Natural products have played a key role in pharma research, as many medicines are either natural products or derivatives thereof. Indeed, it is estimated that about $40 \%$ of all medicines is either natural products or their semisynthetic derivatives [4].

Clinical, pharmacological, and chemical studies of these traditional medicines, which were derived predominantly from plants, were the basis of most early medicines such as aspirin, digitoxin, morphine, quinine, and pilocarpine [5].

Despite competition from other drug discovery methods, natural products are still providing their fair share of new clinical candidates and drugs [5]. These compounds were still a significant source of new drugs, especially in the anticancer, antihypertensive, anti-infectives, immunosuppression, and neurological disease therapeutic areas, and some of them have since progressed further into clinical trials or onto the market [5].

Therefore, in addition to being a proven and important source of drug leads, natural products derived drugs also contribute significantly to the profitability of many companies.

Natural products research continues to explore a variety of lead structures, which may be used as templates for the development of new drugs by the pharmaceutical industry [2]. These approved substances, representative of very wide chemical diversity, continue to demonstrate the importance of compounds from natural sources in modern drug discovery efforts [6].

In addition, natural products, containing inherently large-scale structural diversity than synthetic compounds, have been the major resources of bioactive agents and will continually play as protagonists for discovering new drugs [7]. Drug discovery from medicinal plants has mainly relied on biological activity guided isolation methods which have led to the discovery of important drugs [1].

The place of natural products in the therapeutic arsenal was rigorously discussed and analyzed [1]. A natural product is a chemical compound or substance produced by a living organism-found in nature that usually has a pharmacological or biological activity for use in pharmaceutical drug discovery and drug design. This product can be considered as such even if it can be prepared by total synthesis. It's may be extracted from tissues of terrestrial plants, marine organisms or microorganism fermentation broths. A crude (untreated) extract from any one of these sources typically contains novel, structurally diverse chemical compounds.

Not all natural products can be fully synthesized and many natural products have very complex structures that are too difficult and expensive to synthesize on an industrial scale. These include drugs such as penicillin, morphine, and formerly paclitaxel. Such compounds can only be harvested from their natural source-a process which can be tedious, time consuming, and expensive, as well as being potentially unsustainable for the resource. Furthermore, the number of structural analogues that can be obtained from harvesting is severely limited.

A further problem is that isolates often work differently than the original natural products which have synergies and may combine, say, antimicrobial compounds with compounds that stimulate various pathways of the immune system.

Many higher plants contain novel metabolites with different biological properties. However, in the developed world almost all clinically used chemotherapeutics have been produced by in vitro chemical synthesis. Exceptions, like taxol and vincristine, were structurally complex metabolites that were difficult to synthesize in vitro. Many non-natural, synthetic drugs cause several side effects that were not acceptable except as treatments of last resort for terminal diseases such as cancer. The metabolites discovered in medicinal plants and other natural 
products may avoid the side effect of synthetic drugs, because they must accumulate within living cells.

Semi-synthetic procedures can sometimes get round these problems. This often involves harvesting a biosynthetic intermediate from the natural source, rather than the final (lead) compound itself. The intermediate could then be converted to the final product by conventional synthesis. This approach can have two advantages. First, the intermediate may be more easily extracted in higher yield than the final product itself. Second, it may allow the possibility of synthesizing analogues of the final product. The semi-synthetic penicillins are an illustration of this approach. Another recent example is that of paclitaxel which was manufactured by extracting 10-deacetylbaccatin from the needles of the yew, then carrying out a four-stage synthesis.

Drug discovery from natural products has reclaimed the attention of the Pharma industry and is on the verge of a comeback due to new technological inputs that promise better returns on investment.

Natural products, particularly, microbial and plant products in their native form have been associated with mankind since ancient times. These have played a vital role in the discovery of new chemical entities for drug discovery. Drugs derived from natural sources also served as drug leads suitable for optimization by synthetic means. For certain therapy areas, such as antimicrobials, anticancer antihypertensive and anti-inflammatory drugs, the number was even higher the most of all approved small molecule new chemical entities were derived from nature. The latter is also recognized as important source of biodiversity [8].

In medical science, the loss of species could reduce the opportunity for treatment of diseases through the loss of medical models and new medicines as a result of reduction of the availability of natural products which have potential medicinal properties.

The different approaches used in selecting and screening natural products as candidates for drug discovery and for drug development were rigorously discussed [1]. Contribution of some of these natural compounds in drugs discovery are also reported in the literature $[1,2]$.

\section{The Successful of Natural Products in Drug Discovery}

An integrative approach by combining the various discovery tools and the new discipline of integrative biology will surely provide the key for success in natural product drug discovery and development. Natural products can be predicted to remain an essential component in the search and development for new, safe and economical medicaments. Pharmaceutical industry must awaken in this case to change its mindset and reorient its resources towards the natural product-based drug discovery programs.

According to Lutz [9], natural products not only complement synthetic molecules, they also exhibit drugrelevant features unsurpassable by any synthetic compound. One key feature of natural products is their enormous structural and chemical diversity. In fact, about $40 \%$ of the chemical scaffolds found in natural products are absent in today's medicinal chemistry, and therefore complementary to synthetically produced molecules. Most possibly this is one of the reasons for their historical success in drug discovery, with $45 \%$ of today's bestselling drugs originating from natural products or their derivatives.

Another important advantage of natural products is that they have a biological history. Biosynthesis of natural products involves repeated interaction with modulating enzymes, and the actual biological function of many natural products comprises binding to other proteins. Thus, the ability of natural products to interact with other molecules, an indispensable prerequisite to making an effective drug, might be considered as biologically validated. It is an unsurprising, but often overlooked, fact that many natural products exhibit advanced binding characteristics compared with synthetics. Most probably, the sterically more complex structure of natural products contributes to this [9].

Furthermore, natural products have higher molecular weights; incorporate fewer nitrogen, halogen, or sulfur atoms but more oxygen atoms; and are sterically more complex, with more bridgehead tetrahedral carbon atoms, rings, and chiral centers.

The success of natural products is related to the forces of natural products chemistry, molecular and cellular biology, synthetic and analytical chemistry, biochemistry, and pharmacology to exploit the vast diversity of chemical structures and biological activities of these products.

Moreover, the exploration of structural chemical databases comprising a wide variety of chemotypes, in conjunction with databases on target genes and proteins, will facilitate the creation of new chemical entities through computational molecular modeling for pharmacological evaluation [10].

In industrialized nations at the present time, some fifty percent of all prescribed drugs are derived or synthesized from natural products, the only available sources for which are animals, marine, plants, and micro-organisms. It is considered that because of the structural and biological diversity of their constituents, terrestrial plants offer a unique and renewable resource for the discovering of potential new drugs and biological entities $[1,8]$.

There is no evidence that natural products, containing inherently large-scale structural diversity than synthetic compounds, have been the principal resources of bio- 
logically active agents and will continually act as protagonists for the discovery of new drugs.

In fact, the value of natural products in the treatment and prevention of human diseases can be assessed, according to Chin et al. [6] using three criteria: 1) the rate of introduction of new chemical entities of a wide structural diversity, including their application as templates for semi synthetic and total synthetic modification; 2) the number of diseases treated or prevented by these substances; and 3) their frequency of use in the treatment of diseases.

Natural products are important sources for new drugs and are also good lead compounds suitable for further modification during drug development. The large proportion of natural products in drug discovery has stemmed from the diverse structures and the intricate carbon skeletons of natural products. Since secondary metabolites from natural sources have been elaborated within living systems, they are often perceived as showing more "drug-likeness and biological friendliness than totally synthetic molecules", making them good candidates for further drug development [6].

A potential explanation beyond the success of natural products as drugs is the classification of natural compounds as so-called privileged structures. This concept is based on the fact that chemical agents produced by living organisms (particularly the secondary metabolites) have evolved throughout millenniums under the evolutionary pressure, and are therefore more likely to have a specific biological activity than "randomly" assembled, manmade synthetic chemicals. Despite the enormous potential, only a minor part of globe's living species has ever been tested for any bioactivity. For instance, approximately only $10 \%$ of all existing plant species has been assayed, and in the case of microbes the value is even lower.

In medicine, biotechnology and pharmacology, drug discovery is the process by which drugs are discovered and/or designed. In the past most drugs have been discovered either by identifying the active ingredient from traditional remedies or by serendipitous discovery. A new approach has been to understand how disease and infection are controlled at the molecular and physiological level and to target specific entities based on this knowledge.

The process of drug discovery is so long involves the identification of candidates, synthesis, characterization, screening, and assays for therapeutic efficacy. Once a compound has shown its value in these tests, it will begin the process of drug development prior to clinical trials [1].

\subsection{Screening and Design}

Advancement in the knowledge of molecular mecha- nisms, cellular biology and genomics not only increased the number of molecular targets but also demanded shorter drug discovery timelines. The advent of newer combinatorial techniques of synthesis and computational methods, with the incongruity of natural products to keep pace with the ever growing competition for novel classes of drugs at a faster rate, has prompted pharmaceutical industries to look towards synthetic chemical libraries. Though pharmaceutical industry appreciates the role of nature as the chief architect of natural products' libraries and respect the science therein, they fear carrying out research in the area. However, the rethinking on fresh strategies is on the verge of gaining prominence due to disappointing results of combinatorial chemistry and high throughput screening (HTS) in delivering potent chemical entities.

The old laborious processes involved in the extraction and isolation were not capable of generating the numbers which were required to keep pace with the HTS requirements. Actinomycetes and fungi are used as screening sources by all or almost all organizations that conduct natural product-based drug discovery (NPDD) programs. Bacteria, plants, and medicinal herbs come next. Some apply invertebrates and microalgae to screening. Insects are rarely exploited. But some seek microbes from unusual environments. Since initial natural products are rarely launched unaltered as a drug, unreasonable expectations on financial investment will prevent many countries from being involved in the drug discovery process.

Pharmacognosy provides the tools to identify select and process natural products destined for medicinal use. Usually, the natural product compound has some form of biological activity and that compound is known as the active principle-such a structure can act as a lead compound (not to be confused with compounds containing the element lead). Many of today's medicines are obtained directly from a natural source.

The process of finding a new drug against a chosen target for a particular disease usually involves HTS, where large libraries of chemicals are tested for their ability to modify the target.

Another important function of HTS is to show how selective the compounds are for the chosen target. The ideal is to find a molecule which will interfere with only the chosen target, but not other, related targets. To this end, other screening runs will be made to see whether the "hits" against the chosen target will interfere with other related targets-this is the process of cross-screening. Cross-screening is important, because the more unrelated targets a compound hits, the more likely that off-target toxicity will occur with that compound once it reaches the clinic.

It is very unlikely that a perfect drug candidate will emerge from these early screening runs. It is more often 
observed that several compounds are found to have some degree of activity, and if these compounds share common chemical features, one or more pharmacophores can then be developed. At this point, medicinal chemists will attempt to use structure-activity relationships to improve certain features of the lead compound.

This process will require several iterative screening runs, during which, it is hoped, the properties of the new molecular entities will improve, and allow the favored compounds to go forward to in vitro and in vivo testing for activity in the disease model of choice.

While HTS is a commonly used method for novel drug discovery, it is not the only method. It is often possible to start from a molecule which already has some of the desired properties. Such a molecule might be extracted from a natural product or even be a drug on the market which could be improved upon. Other methods, such as virtual high throughput screening, where screening is done using computer-generated models and attempting to “dock” virtual libraries to a target, are also often used.

Another important method for drug discovery is drug design, whereby the biological and physical properties of the target are studied, and a prediction is made of the sorts of chemicals that might fit into an active site. Novel pharmacophores can emerge very rapidly from these exercises.

Once a lead compound series has been established with sufficient target potency and selectivity and favorable drug-like properties, one or two compounds will then be proposed for drug development. The best of these is generally called the lead compound, while the other will be designated as the "backup".

Combinatorial chemistry may simply have increased the volume of haystack in which the needle is hidden. Natural compounds certainly have biological activity in their original locations. However, natural product research needs long and deep experience, particularly in taxonomy, which cannot be built up in one day. If this know-how is abandoned, it will never be recovered in the future. One of the solutions will be out-sourcing the activity, because a number of technologically advanced biotech ventures will play a key role of NPDD.

The modern natural product research is undergoing a revolution due to recent advancements in combinatorial biosynthesis, microbial genomics and screening processes. Moreover, access to hyphenated techniques like Liquid Chromatography-Mass Spectrometry, Liquid Chromatography-Nuclear Magnetic Resonance have raised the hope of drastically reducing the time and cost involved in natural product research by using dereplication processes that are combination of techniques to avoid the already reported compounds.

The unique molecular diversity of natural compounds can be leveraged in the design of combinatorial libraries to improve their inherent biological activity or drug-like properties. This can be performed by semi-synthetic modification of the parent molecule or by fully synthetic methods after crucial structural elements required for biological activity are defined from the libraries and refined by computer-assisted drug designing methods. In comparison to the past, more specific targets, efficient in vitro as well as in vivo models are available and can be utilized. Current strategies in natural product research involve a multifaceted approach combining botanical, phytochemicals, biological and molecular techniques.

In a paradigm shift from discovery of single bioactive molecules, multi-constituent mainstay of bioactive extracts can be emphasized for synergistic and antagonistic studies at cellular and molecular levels. Of the 250,000 species of higher terrestrial plants in existence, only 5 to 15 percent are estimated to have been chemically and pharmacologically investigated in systematic fashion.

Today, the perceivable threat of extinction of biodiversity due to global warming and other environmental reasons is also very high. Thus, there is an urgent need to identify the indigenous natural resources to study them in detail for use in drug discovery. Moreover, sustainable supply of medicinal plants is essential for practicing traditional medicine as well as natural product drug discovery.

The pharmaceutical industry has to come forward in utilizing the knowledge available in traditional medicines. These later may provide cure for different types of diseases and disorders but need scientific validation. In order to focus the research on traditional medicines to serve national interests, the first priority is to assess the therapeutic quality of herbal medicines objectively since the dividing line between the modern therapies and traditional therapies remains imprecise.

The assessment of quality can be made easier if distinction is drawn between rational and empirical medication taking into account marked psychodynamic effects associated not only with the active substances, but also with their typical indications. It is satisfying to note that companies have started their efforts for using the traditional knowledge in the development of their formulations as well as in drug discovery programs. The formulations that will be manufactured in these companies use Good Manufacturing Practices and Best Quality Practices to ensure standardized, safe and effective herbs as well as finished phytomedicines that can be made popular like the Chinese medicines all over the world.

The therapeutic areas of inflammation, metabolic diseases and obesity may receive more attention for the development of natural products. Inflammation process is considered to be the root cause of almost all the diseases including cancer at the molecular level. Lifestyle diseases like obesity and metabolic disorders are silent 
killers which have gone unnoticed so far. A huge amount of information is already available in traditional medicine about their cures. Many plants and their secondary metabolites have already been researched for these areas and the pharmaceutical industry should take steps in utilizing this valuable information.

\subsection{Chemical Diversity of Natural Products}

It is not uncommon for natural products to have complex molecular structures, with cyclic semi-rigid scaffolds, several chiral centers, more than five H-bond donors, more than ten $\mathrm{H}$-bond acceptors, more than five rotatable C-C bonds, a large polar surface area, and a molecular weight above 500. While this may lead to moderate levels of bioavailability and corresponding dosage regimens to achieve the required efficacy, there may be a latent advantage in such complex structures, as has been previously hypothesized. This complexity has occasionally been thought to be a constraint for the total chemical synthesis of natural products-based libraries in a given time and resource frame [4].

Chemical diversity in nature is based on biological and geographical diversity, so researchers travel around the world obtaining samples to analyze and evaluate in drug discovery screens or bioassays. This effort to search for natural products is known as bioprospecting.

On the other hand, some medicines are developed from a lead compound originally obtained from a natural source. This means the lead compound can be produced by total synthesis, or can be a starting point (precursor) for a semi-synthetic compound, or can act as a template for a structurally different total synthetic compound.

This is because most biologically active natural product compounds are secondary metabolites with very complex structures. This has an advantage in that they are extremely novel compounds but this complexity also makes many lead compounds' synthesis difficult and the compound usually has to be extracted from its natural source-a slow, expensive and inefficient process. As a result, there is usually an advantage in designing simpler analogues.

Historically, most drugs have been derived from natural products, but there has been a shift away from their use with the increasing predominance of molecular approaches to drug discovery. Nevertheless, their structural diversity makes them a valuable source of novel lead compounds against newly discovered therapeutic targets. Technical advances in analytical techniques mean that the use of natural products is easier than before [11].

It has long been recognized that natural-product structures have the characteristics of high chemical diversity, biochemical specificity and other molecular properties that make them favorable as lead structures for drug dis- covery, and which serve to differentiate them from libraries of synthetic and combinatorial compounds [12].

Moreover, natural products typically have a greater number of chiral centers and increased steric complexity than either synthetic drugs or combinatorial libraries [12].

In addition, natural products differ significantly from synthetic drugs and combinatorial libraries in the ratio of aromatic ring atoms to total heavy atoms (lower in natural products), number of solvated hydrogen-bond donors and acceptors (higher in natural products) and by greater molecular rigidity. Natural-product libraries also have a broader distribution of molecular properties such as molecular mass, octanol-water partition coefficient and diversity of ring systems compared with synthetic and combinatorial counterparts. Indeed, less than one-fifth of the ring systems found in natural products are represented in current trade drugs [12].

\subsection{Isolation and Purification}

If the lead compound (or active principle) is present in a mixture of other compounds from a natural source, it has to be isolated and purified. The ease with which the active principle can be isolated and purified depends very much on the structure, stability, and quantity of the compound. In past, it was not possible until the development of new experimental procedures such as freeze drying and chromatography that the successful isolation and purification of penicillin and other natural products became feasible.

\subsection{Identification of Biologically Active Material}

Two main approaches exist for the finding of new bioactive chemical entities from natural sources; random collection and screening of material, or exploitation of ethnopharmacological knowledge in the selection [1]. The former approach bases itself on the fact that only a very small part of globes' biodiversity has ever been tested for any biological activity, and on the other hand, particularly organisms living in a species-rich environment need to evolve defense and competition mechanism to survive. Thus, collection of plant, animal and microbial samples from rich ecosystems may give rise to isolation of novel biological activities. One example of a successful use of this strategy is the screening for antitumor agents, performed by National Cancer Institute in USA started in 1960s. Cytostate paclitaxel (taxoid) was identified during this campaign from Pacific yew tree Taxus brevifolia. Paclitaxel showed antitumor activity with previously unknown mechanism (stabilization of microtubules) and is now approved for clinical use for the treatment of lung, breast and ovary cancer, as well as for Kapos sarcoma.

Besides random selection, the selection of starting 
material may be done by collecting knowledge on use of plants and other natural products as herbal medicines and thereby get an idea of potential biological activities. It is worth remembering that a major part of earth's population still relies on nature-derived drugs as their only medication. Ethnobotany, the study of the use of plants in the society, and particularly ethnopharmacology, an area inside ethnobotany focused on medical use of plants, may therefore provide invaluable information, as illustrated by the example of artemisinin, an antimalarial agent from sweet wormtree Artemisiae annua, used in Chinese medicine since 200 DC and nowadays in use against multiresistant malarial protozoa Plasmodium falsiparum.

\subsection{Structure-Activity and Structure-Property Relationships of Natural Products}

One of the distinguishing features of natural products is their frequent occurrence as suites or complexes of structurally related analogues. The biological significance of this expressed molecular diversity is unclear, particularly when the suite contains members (often major) that seem to lack biological activity or function. Essentially, why would an organism expend the resources needed to synthesize many analogues of a molecule for which there is but a single purpose? One possible answer to this question is furnished by the "screening hypothesis", which is based on the proposition that biological activity is a rare property for any molecule to possess, and that there might be a selective advantage to an organism if it can generate and retain chemical diversity at a low cost. The existence of congeners of a natural product series might therefore be the consequence of an organism's need to generate its own chemical diversity to optimize the activity of its secondary metabolites, essentially doing its own structure-activity relationship (SAR) optimization [12].

\subsection{Development of New Technologies in Pharmaceutical Discovery Research: Modern Methods of Drug Discovery}

The success of drugs derived from nature added a new driving force to screening for novel, biologically active metabolites from these products. Since the 90s, new technologies such as combinatorial chemistry, highthroughput screening, bioinformatics, proteomics and genomics have emerged, and are being integrated widely in the field of pharmaceutical discovery research. These technologies have enormous potential to make use of the chemical diversity of natural products. Other techniques have only been developed in the last years and continue to evolve rapidly. These include molecular diversity, compound library design, protein 3D-structures, NMRbased screening, 3D QSAR in modern drug design, physicochemical concepts, and computer-aided prediction of drug toxicity and metabolism.

During that period, technologies for drug discovery advanced and diversified greatly. NPDD activities compete rather strongly with HTS, combinatorial chemistry and genomics. New approaches to improve and accelerate the joint drug discovery and development processes are expected to take place mainly from the innovation in drug target elucidation and lead structure discovery. Powerful new technologies are revolutionizing drug discovery.

When using natural products to support HTS, discovery programs must include effective technologies to support the acquisition and inventory of the natural products sources. A critical element in this process is information handling. Information handling requirements at every stage of this process will grow, and computer technology is available to meet the demand.

With the advance of screening technologies, it is now possible to evaluate upwards of 100,000 test substances per week against several different targets. The required test substance quantities are as low as a few micrograms per assay. In keeping with these trends, natural products acquisition programs are now scoped to collect large numbers of diverse tissue samples, but only to collect small wet weights. This format allows collectors to provide large numbers of samples for screening, and they rely on recollection for follow-on studies. This means that discovery programs actively testing natural products must track a greater number of samples. In addition, information on known natural products chemistry must be considered in an effort to contain the costs of follow-on studies.

General information handling system requirements are recollection and dereplication. These requirements share a common feature. The latitude-longitude location of a source organism collection can be easily obtained using satellite navigation technologies and placed on a map. The position of past collections can be similarly mapped; however, the accuracy of these data will vary. It is possible to map these positions in a geographic information system (GIS) where computer-based mapped data are linked to traditional text/field database records. Using a comprehensive dataset of natural products chemical discoveries within a GIS would allow investigators to easily visualize the spatial relationships between collection points to support decisions. Furthermore, expeditions for new acquisition efforts could effectively be planned to target specific regions based on existing information, and to avoid duplicating past collections.

One of the most powerful features of a comprehensive information management system that supports natural products acquisition programs is use of spatial data and GIS technology. GIS technology allows the system to 
step beyond the requirements for a normalized data model in a relational database design. GIS allows for addition of those data, which have a spatial element, as an overlay. Importantly, spatial data can be selectively added on a need to know basis, and, most importantly, addition of these varied data does not affect the logical design and data model.

HTS is an information-intensive industry. The breadth of existing information that is necessary to consider in the discovery process, the increasing number of samples submitted for testing and the evolving discovery screen models all contribute to a growing dataset. Currently available technologies can meet the information handling requirements for natural products acquisition programs. These technologies, however, must be effectively realized for an application. For example, GIS technology is a powerful tool that is typically delivered with extended capabilities that reach beyond the described general requirements for this application. In developing an effective solution, the GIS capability must be delivered in a customized format that provides only those features needed by the natural products investigator-again, these technologies must be realized for the application.

These information management requirements, however, can become assets that are used to guide the collection effort and enhance the probability of success. Screening natural products extracts does not necessarily require a random approach. Examples of structure/activity relationships are beginning to emerge for a variety of biological assays. Published information on natural products chemistry and related biological activity can guide a collection effort to target, but not duplicate collections from, organism groups with known properties. A contemporary drug discovery program requires advanced capabilities for information handling.

The use of mass spectrometry (MS) and genomics-derived sequence databases for protein characterization is briefly discussed, as are new directions such as HPLC and capillary electrophoresis for separation, proteomic diagnostics, and target discovery and validation.

Bioinformatics is discussed next, as a "theoretical, computer-based science dedicated to the extrapolation of biological knowledge from biological information”. Bioinformatics today is mainly the filtering and assessing of likely drug target candidates, especially targets derived from genomic applications. Genomics produces huge numbers of sequences, but those genetic sequences must be examined to identify the regions of similarity and/or identity and thus of possible pharmaceutical interest.

Recent technological advances that help to address these issues, coupled with unrealized expectations from current lead-generation strategies, have led to a renewed interest in natural products in drug discovery. This latter should be utilized as one of genomics-based drug dis- covery strategies.

However, despite the rise of combinatorial chemistry as an integral part of lead discovery process, the natural products still play a major role as starting material for drug discovery.

Natural product-based drug discovery (NPDD) activities compete rather strongly with high-throughput screening (HTS), combinatorial chemistry and genomics. Recently, Genomics and CBD have made a great impact on NPDD, whereas emerging new biotech ventures based on NPDD and biological resource centers are expected to play a key role in this field.

These technologies have enormous potential to make use of the chemical diversity of natural products. At the outset it is made clear that none of these methods are a magic cure-all that will instantly shave years off the drug discovery cycle, but instead are complementary tools with different strengths and weaknesses, which must be applied appropriately and synergistically.

\subsubsection{Combinatorial Chemistry}

Combinatorial chemistry generates larger libraries but the compounds therein are relatively simple planar molecules contrary to the natural products' pool that gives a much higher hit-rate in high throughput screening with high chemical diversity. Further, improvements in isolation, purification and characterization procedures have fastened the output of natural product research, thereby reviving the interest of the pharmaceutical industry.

Combinatorial chemistry has evolved from its early focus as a random strategy for generating molecular diversity into a powerful design technology for developing and optimizing drug candidates.

Increasing pressure to identify, optimize, develop, and commercialize novel drugs more rapidly and more costeffectively has led to an urgent demand for technologies that can reduce the time to market for new products. Molecular diversity, of both natural and synthetic materials, provides a valuable source of compounds for identifying and optimizing new drug leads. Through the rapidly evolving technology of combinatorial chemistry, it is now possible to produce libraries of small molecules to screen for novel bioactivities. This powerful new technology has begun to help pharmaceutical companies to find new drug candidates quickly, save significant dollars in preclinical development costs, and ultimately change their fundamental approach to drug discovery.

Comprising the work of the leading authorities in the area of molecular diversity and combinatorial chemistry, combinatorial chemistry and molecular diversity in drug discovery highlights the critical concepts and issues involved in implementing combinatorial chemistry to create chemical libraries.

As above mentioned, combinatorial chemistry was a 
key technology enabling the efficient generation of large screening libraries for the needs of high-throughput screening. However, now, after two decades of combinatorial chemistry, it has been pointed out that despite the increased efficiency in chemical synthesis, no increase in lead or drug candidates have been reached. This has led to analysis of chemical characteristics of combinatorial chemistry products, compared to existing drugs and/or natural products. The chemo-informatics concept chemical diversity, depicted as distribution of compounds in the chemical space based on their physicochemical characteristics, is often used to describe the difference between the combinatorial chemistry libraries and natural products. The synthetic, combinatorial library compounds seem to cover only a limited and quite uniform chemical space, whereas existing drugs and particularly natural products, exhibit much greater chemical diversity, distributing more evenly to the chemical space. The most prominent differences between natural products and compounds in combinatorial chemistry libraries are the number of chiral centers (much higher in natural compounds), structure rigidity (higher in natural compounds) and number of aromatic moieties (higher in combinatorial chemistry libraries). Other chemical differences between these two groups include the nature of heteroatoms ( $\mathrm{O}$ and $\mathrm{N}$ enriched in natural products, and $\mathrm{S}$ and halogen atoms more often present in synthetic compounds), as well as level of non-aromatic unsaturation (higher in natural products). As both structure rigidity and chirality are both well-established factors in medicinal chemistry known to enhance compounds specificity and efficacy as a drug, it has been suggested that natural products compare favorable to today's combinatorial chemistry libraries as potential lead molecules.

\subsubsection{High-Throughput Screening}

High-Throughput Screening (HTS) is a standard method for hit discovery for scientific experimentation especially used in drug discovery and relevant to the fields of biology and chemistry [13].

Using robotics, data processing and control software, liquid handling devices, and sensitive detectors, HTS allows a researcher to quickly conduct millions of biochemical, genetic or pharmacological tests. Through this process one can rapidly identify active compounds, antibodies or genes which modulate a particular biomolecular pathway. The results of these experiments provide starting points for drug design and for understanding the interaction or role of a particular biochemical process in biology.

In essence, HTS uses a brute-force approach to collect a large amount of experimental data-usually observations about how some biological entity reacts to exposure to various chemical compounds - in a relatively short time. A screen, in this context, is the larger experiment, with a single goal (usually testing a scientific hypothesis), to which all this data may subsequently be applied.

A screening facility typically holds a library of stock plates, whose contents are carefully catalogued, and each of which may have been created by the lab or obtained from a commercial source. These stock plates themselves are not directly used in experiments; instead, separate assay plates are created as needed. An assay plate is simply a copy of a stock plate, created by pipetteing a small amount of liquid (often measured in nanoliters) from the wells of a stock plate to the corresponding wells of a completely empty plate.

Automation is an important element in HTS's usefulness. A specialized robot is often responsible for much of the process over the lifetime of a single assay plate, from creation through final analysis. An HTS robot can usually prepare and analyze many plates simultaneously, further speeding the data-collection process. HTS robots currently exist which can test up to 100,000 compounds per day.

HTS is a relatively recent innovation, made lately feasible through modern advances in robotics and highspeed computer technology. It still takes a highly specialized and expensive screening lab to run an HTS operation, however, so in many cases a small-to-moderately sized research institution will use the services of an existing HTS facility rather than set up one for it.

According to Dietzman [14], when using natural products to support High Throughput Screening (HTS), discovery programs must include effective technologies to support the acquisition and inventory of the natural products sources. A critical element in this process is information handling. Information handling requirements at every stage of this process will grow, and computer technology is available to meet the demand.

With the advance of screening technologies, it is now possible to evaluate upwards of 100,000 test substances per week against several different targets. The required test substance quantities are as low as a few micrograms per assay. In keeping with these trends, natural products acquisition programs are now scoped to collect large numbers of diverse tissue samples, but only to collect small wet weights. This format allows collectors to provide large numbers of samples for screening, and they rely on recollection for follow-on studies. This means that discovery programs actively testing natural products must track a greater number of samples. In addition, information on known natural products chemistry must be considered in an effort to contain the costs of follow-on studies.

When a natural product registers as a confirmed "hit" in a discovery screen, the people involved simply want to know: what is it, where did it come from, is it novel and 
how to get more. The general information handling system requirements, therefore, fall into two categories: recollection, and dereplication.

Recollection must be a cornerstone of any natural products acquisition program; you must be able to go back and get more of the source organism for follow-on studies. Dereplication against the growing number of known chemical compounds is becoming increasingly important for discovery screens. Based upon initial chemical characterization of an active fraction(s), a chemical substructure-based database search can be performed to compare with, and dereplicate against, known chemical compounds. Laboratory chemists are at a tremendous advantage when they can generate a list of possible chemical structures to compare with an extract containing unknown compounds. A chemist can then quickly evaluate the possibility for the presence of known compounds and will be able to make an informed decision regarding the interest in the extract as a source of novel bioactive compounds. This rapid process can save the costs of follow-on isolation and structural elucidation studies and increase a program's efficiency.

\subsubsection{Bioinformatics, Proteomics and Genomics}

Proteomics include technologies for protein mapping (separating, distinguishing and quantifying the proteins present in individual samples), and techniques for identifying specific proteins and characterizing their complete structure and functional role. The main protein mapping technology in use today is two-dimensional polyacrylamide gel electrophoresis (2D-PAGE), which can resolve up to 2000 proteins on a single gel—significantly better than other separation techniques, but still not the 6000 proteins expressed by typical tissues.

According to Harvey [11], with recent technological developments in molecular biology, instrumentation and information technology, screening of compounds can be conducted at throughputs that could not have been imagined, even a few years ago. The availability of molecular targets, the ability to engineer such targets into simple reporter systems such as yeasts, and the use of robotics to handle the samples and conduct the assays make random screening of chemical diversity a very attractive approach to the discovery of novel activity. The techniques of molecular cloning provide the possibility of deriving an understanding of physiological processes at the molecular level. Currently, over 250 gene products relating to major neurotransmitters are known, and hundreds of different subtypes of ion channels have also been characterized genetically. There has been a similar increase in the understanding of intracellular signaling pathways, opening up the possibility of many new target sites for drugs.

The drug discovery process is becoming more and more complex and capital-intensive, and such companies remain "target rich" but "lead poor", with lead discovery as a greater bottleneck. In such a situation, industrialization of drug discovery process is underway. Although highthroughput screening (HTS) and combinatorial chemical synthesis are explored with great hope, general experience tells us that in most companies the investments in these technologies have not reaped rewards in new lead discovery as expected.

Powerful new technologies such as HTS and combinatorial chemistry are revolutionizing drug discovery. But natural products still offer unmatched structural variety, especially as new environmental niches are explored, and their usefulness can be further extended by engineering the proteins that produce them and using them to probe biological pathways [15].

According to Lutz [9], the human genome has provided the pharmaceutical industry with innumerable starting points for new drug discovery approaches. And yet, the expected flood of new chemical entities has failed to materialize.

On the other hand, the sequencing of the human genome and numerous pathogen genomes has resulted in an unprecedented opportunity for discovering potential new drug targets. Chemogenomics has appeared as a new technology to initiate target discovery by using active compounds as probes to characterize proteome functions. Natural products are the ideal probes for such research. Binding affinity fingerprint is a powerful chemogenomic descriptor to characterize both small molecules and pharmacologically relevant proteins [7].

\section{Future Perspectives}

Combinatorial chemistry may simply have increased the volume of haystack in which the needle is hidden. Natural compounds certainly have biological activity in their original locations. However, natural product research needs long and deep experience, particularly in taxonomy, which cannot be built up in one day. If this knowhow is abandoned, it will never be recovered in the future. One of the solutions will be out-sourcing the activity, because a number of technologically advanced biotech ventures will play a key role of NPDD.

Biodiversity and genomics are two key words governing bioscience in the 21st century. Biological diversity is increasingly important in this field. Industries need to adapt to the new regulations and have clear visions of what their needs are for biological resources as tools for innovation. On the other hand, organizations with title and access to resources need to find ways to facilitate sustainable use of their biological resources if they really want to participate in and benefit from the drug discovery process. Everyone should understand that even though a 
biological resource may have great intrinsic value as a component of an ecosystem and a reservoir of genetic information, its monetary value might be minimal until a utility is discovered and developed. To realize that utility and to share in the benefits, organizations should be prepared not only to participate actively in the discovery process, but also to share the financial risks.

\section{Conclusions}

There is no doubt that plants are among the most perfect "natural laboratories" for the synthesis of various mole- cules ranging from simple skeleton to highly complex chemical structures. If secondary metabolites are compared with randomly synthesized compounds, natural metabolites are superior in terms of biological and pharmacological activities. Present drug discovery from medicinal plants has mainly relied on biological activity guided isolation methods, which, for example, have led to the isolation, identification and the discovery of important drugs [16,17] (Tables 1-3). Drug discovery begins with attempts to find a molecule that causes a specific biological response. In the highly competitive

Table 1. Drugs derived from plants with their ethnomedical correlations and sources [16,17].

\begin{tabular}{|c|c|c|}
\hline Drug & Action or clinical use & Plant source \\
\hline Acetyldigoxin & Cardiotonic & Digitalis lanata Ehrh. \\
\hline Adoniside & Cardiotonic & Adonis vernalis L. \\
\hline Aescin & Anti-inflammatory & Aesculus hippocastanum L. \\
\hline Aesculetin & Antidysentery & Fraxinus rhynchophylla Hance \\
\hline Agrimophol & Anthelmintic & Agrimonia eupatoria L. \\
\hline Ajmalicine & Circulatory disorders & Rauvolfia serpentina (L.) Benth ex. Kurz \\
\hline Allyl isothiocyanate & Rubefacient & Brassica nigra (L.) Koch \\
\hline Andrographolide & Bacillary dysentery & Andrographis paniculata Nees \\
\hline Anisodine & Anticholinergic & Anisodus tanguticus (Maxim.) Pascher \\
\hline Arecoline & Anthelmintic & Areca catechu L. \\
\hline Asiaticoside & Vulnerary & Centella asiatica (L.) Urban \\
\hline Atropine & Anticholinergic & Atropa belladonna L. \\
\hline Berberine & Bacillary dysentery & Berberis vulgaris L. \\
\hline Bergenin & Antitussive & Ardisia japonica $\mathrm{Bl}$. \\
\hline Bromelain & Anti-inflammatory; proteolytic agent & Ananas comosus (L.) Merrill \\
\hline Caffeine & CNS stimulant & Camellia sinensis (L.) Kuntze \\
\hline$(+)$-Catechin & Haemostatic & Potentilla fragaroides L. \\
\hline Cocaine & Local anaesthetic & Erythroxylum coca Lamk. \\
\hline Codeine & Analgesic: Antitussive & Papaver somniferum L. \\
\hline Colchicine & Antitumor agent; antigout; anti-inflammatory & Colchicum autumnale L. \\
\hline Convallotoxin & Cardiotonic & Convallaria majalis L. \\
\hline Curcumin & Choleretic & Curcuma longa L. \\
\hline Cynarin & Choleretic & Cynara scolymus L. \\
\hline Danthron & Laxative & Cassia spp. \\
\hline Deserpidine & Antihypertensive; tranquilizer & Rauvolfia canescens L. \\
\hline
\end{tabular}




\section{Continued}

\begin{tabular}{|c|c|c|}
\hline Deslanoside & Cardiotonic & Digitalis lanata Ehrh. \\
\hline Digitalin & Cardiotonic & Digitalis purpurea $\mathrm{L}$. \\
\hline Digitoxin & Cardiotonic & Digitalis purpurea $\mathrm{L}$. \\
\hline Digoxin & Cardiotonic & Digitalis lanata Ehrh. \\
\hline Emetine & Amoebicide; emetic & Cephaelis ipecacuanha (Brotero) A. Richard \\
\hline Ephedrine & Sympathomimetic & Ephedra sinica Stapf. \\
\hline Etoposide & Antitumour agent & Podophyllum peltatum L. \\
\hline Gitalin & Cardiotonic & Digitalis purpurea $\mathrm{L}$. \\
\hline Glaucaroubin & Amoebicide & Simarouba glauca DC. \\
\hline Glycyrrhizin & Sweetener & Glycyrrhiza glabra L. \\
\hline Gossypol & Male contraceptive & Gossypium spp. \\
\hline Hemsleyadin & Bacillary dysentery & Helmsleya amabilis Diels \\
\hline Hydrastine & Hemostatic; astringent & Hydrastis canadensis L. \\
\hline Hyoscamine & Anticholinergic & Hyoscamus niger L. \\
\hline Kainic acid & Ascaricide & Digenea simplex (Wulf.) Agardh \\
\hline Kawain & Tranquilizer & Piper methysicum Forst. f. \\
\hline Khellin & Bronchodilator & Ammi visnaga (L.) Lamk. \\
\hline Lanatosides A, B, C & Cardiotonic & Digitalis lanata Ehrh. \\
\hline Lobeline & Smoking deterrent; respiratory stimulant & Lobelia inflata L. \\
\hline Monocrotaline & Antitumor agent & Crotolaria sessiliflora L. \\
\hline Morphine & Analgesic & Papaver somniferum L. \\
\hline Neoandrographolide & Bacillary dysentery & Andrographis paniculata Nees \\
\hline Noscapine & Antitussive & Papaver somniferum $\mathrm{L}$. \\
\hline Ouabain & Cardiotonic & Strophanthus gratus Baill. \\
\hline Papain & Proteolytic; mucolytic & Carica papaya L. \\
\hline Papaverine & Sympatholytic musculotropic & Papaver somniferum L. \\
\hline Phyllodulcin & Sweetener & Hydrangea macrophylla (Thunb.) DC \\
\hline Physostigmine & Cholinesterase inhibitor & Physostigma venenosum Balf. \\
\hline Picrotoxin & Analeptic & Anamirta cocculus (L.) W. \& A. \\
\hline Pilocarpine & Parasympathomimetic & Pilocarpus jaborandi Holmes \\
\hline Podophyllotoxin & Condylomata acuminata (Topical anti-viral agent) & Podophyllum peltatum L. \\
\hline Protoveratrines A \& B & Antihypertensive & Veratrum album L. \\
\hline Pseudoephedrine & Sympathomimetic & Ephedra sinica Stapf. \\
\hline Pseudoephedrine, nor- & Sympathomimetic & Ephedra sinica Stapf. \\
\hline Quisqualic acid & Anthelmintic & Quisqualis indica $\mathrm{L}$. \\
\hline Quinine & Antimalaric & Cinchona ledgeriana Moens ex. Trimen \\
\hline Rescinnamine & Antihypertensive; tranquilizer & Rauvolfia serpentina (L.) Benth ex. Kurz \\
\hline
\end{tabular}




\begin{tabular}{|c|c|c|}
\hline Reserpine & Antihypertensive; tranquilizer & Rauvolfia serpentina (L.) Benth ex. Kurz \\
\hline Rhomitoxine & Antihypertensive & Rhododendron molle G. Don \\
\hline Rorifone & Antitussive & Rorippa indica (L.) Hochr. \\
\hline Rotenone & Piscicide & Lonchocarpus nicou (Aubl.) DC. \\
\hline Rotundine & Analgesic; sedative & Stephania sinica Diels \\
\hline Salicin & Analgesic & Salix alba L. \\
\hline Santonin & Ascaricide & Artemisia maritima $\mathrm{L}$. \\
\hline Scillarin A & Cardiotonic & Urginea maritima (L.) Baker \\
\hline Scopolamine & Sedative & Datura metel L. \\
\hline Sennosides A \& B & Laxative & Cassia spp. \\
\hline Silymarin & Antihepatotoxic & Silybum marianum (L.) Gaertn. \\
\hline Stevioside & Sweetener & Stevia rebaudiana Bertoni \\
\hline Strychnine & CNS stimulant & Strychnos nux-vomica L. \\
\hline Teniposide & Antitumor agent & Podophyllum peltatum $\mathrm{L}$. \\
\hline Tetrahydropalmatine & Analgesic; sedative & Corydalis ambigua (Pallas) Cham. \& Schltal. \\
\hline Theobromine & Diuretic; bronchodilator & Theobroma cacao L. \\
\hline Theophylline & Diuretic; bronchodilator & Camellia sinensis (L.) Kuntze \\
\hline Trichosanthin & Abortifacient & Thymus vulgaris L. \\
\hline Tubocurarine & Skeletal muscle relaxant & Chondodendron tomentosum R. \& P. \\
\hline Valepotriates & Sedatifve & Valeriana officinalis L. \\
\hline Vincamine & Cerebral stimulant & Vinca minor L. \\
\hline Xanthotoxin & Leukoderma, vitiligo & Ammi majus L. \\
\hline Yohimbine & Aphrodisiac & Pausinystalia yohimbe (K. Schum.) Pierre \\
\hline Yuanhuacine & Abortifacient & Daphne genkwa Seib. \& Zucc. \\
\hline Yuanhuadine & Abortifacient & Daphne genkwa Seib. \& Zucc. \\
\hline
\end{tabular}

environment of contemporary pharmaceutical research, natural products provide a unique element of molecular diversity and biological functionality, which is indispensable for drug discovery. Moreover, secondary metabolites from plants show a striking structural diversity that supplements chemically synthesized compounds or libraries in drug discovery programs.

To continue to be competitive with other drug discovery methods, natural product research needs to continually improve the speed of the screening, isolation, and structure elucidation processes, as well addressing the suitability of screens for natural product extracts and dealing with issues involved with large-scale compound supply.

There is enormous scope for many countries, with their rich biodiversity, to contribute to this trade. To enhance this, it is important that the cultivation programs of the medicinal plants be carried out using controlled and scientific methods so that these will ensure plant material of desired quality and also help manage the resources effectively. However, the growing demand for medicinal plants is also threatening their existence. For meeting the future needs, cultivation of medicinal plants has to be encouraged.

An integrative approach by combining the various discovery tools and the new discipline of integrative biology will provide the key for success in natural product drug discovery and development.

However, untapped biological resources, "smart screening” methods, robotic separation with structural analysis, metabolic engineering, and synthetic biology offer exciting technologies for new natural product drug discovery. Advances in rapid genetic sequencing, coupled with manipulation of biosynthetic pathways, may provide a vast 
Table 2. Plant-derived drugs and their sources not developed on the basis of ethnomedical information [16,17].

\begin{tabular}{|c|c|}
\hline Drug & Plant source \\
\hline Allantoin & Several plants \\
\hline Anabasine & Anabasis aphylla L. \\
\hline Apomorphine hydrochloride & Papaver somniferum L. \\
\hline Arteether & Artemisia annua L. \\
\hline Benzyl benzoate & Several plants \\
\hline Borneol & Several plants \\
\hline Camphor & Cinnamonum camphora (L.) J.S. Presl \\
\hline Camptothecin & Camptotheca acuminate Decne. \\
\hline Cissampeline & Cissampelos pareira L. \\
\hline Colchicaine amide & Colchicum autumnale L. \\
\hline Demecolcine & Colchicum autumnale L. \\
\hline L-Dopa & Mucuna deeringiana (Bort) Merr. \\
\hline Galanthamine & Lycoris squamigera Maxim. \\
\hline Galantamine hydrobromide & Galanthus nivalis L. \\
\hline Glaucine & Glaucium flavum Crantz \\
\hline Glaziovine & Ocotea glazovii Mez \\
\hline Hesperidin & Citrus spp. \\
\hline Huperzine A & $\begin{array}{c}\text { Huperzia serrata (Thunb. ex Murray) } \\
\text { Trevis. }\end{array}$ \\
\hline Menthol & Mentha spp. \\
\hline Methyl salicylate & Gautheria procumbens L. \\
\hline Nicotine & Nicotiana tabacum L. \\
\hline Nitisinone & Callistemon citrinus Stapf. \\
\hline Nordihydroguaiaretic acid & Larrea divaricata Cav. \\
\hline Pachycarpine & $\begin{array}{c}\text { Sophora pachycarpa Schrenk ex C.A. } \\
\text { Meyer }\end{array}$ \\
\hline Palmatine & Coptis japonica Makino \\
\hline Papaverine & Papaver somniferum L. \\
\hline Pinitol & Several plants \\
\hline Quinidine & Cinchona ledgeriana Moens ex. Trimen \\
\hline Rutin & Citrus spp. \\
\hline Sanguinarine & Sanguinaria canadensis L. \\
\hline Sparteine & Cytisus scoparius (L.) Link. \\
\hline Taxol/paclitaxel & Taxus brevifolia Nutt. \\
\hline Tetrahydrocannabinol & Cannabis sativa L. \\
\hline Tetrandrine & Stephania tetrandra S. Moore \\
\hline Thymol & Thymus vulgaris L. \\
\hline Tiotropium bromide & Atropa belladonna L. \\
\hline Vasicine (peganine) & Adhatoda vasica Nees \\
\hline Vinblastine (Navelbine) & Catharanthus roseus (L.) G. Don \\
\hline Vincristine & Catharanthus roseus (L.) G. Don \\
\hline
\end{tabular}

Table 3. Plant-derived drugs and their sources [16].

\begin{tabular}{|c|c|}
\hline Drug & Plant source \\
\hline Indinavir & Hypericum perforatum L. \\
\hline Nevarapin & Hypericum perforatum L. \\
\hline Cyclosporine & Hypericum perforatum L. \\
\hline Tacrolimus & Hypericum perforatum L. \\
\hline Simvastatin & Hypericum perforatum L. \\
\hline Amitriptylin & Hypericum perforatum L. \\
\hline Midazolam & Hypericum perforatum L. \\
\hline Irinotecan & Hypericum perforatum L. \\
\hline Digoxin & Hypericum perforatum L. \\
\hline Fexofenadine & Hypericum perforatum L. \\
\hline Phenprocoumon & Hypericum perforatum L. \\
\hline Paroxetine & Hypericum perforatum L. \\
\hline Sertralin & Hypericum perforatum L. \\
\hline Trazodon & Hypericum perforatum L. \\
\hline Nefazodon & Hypericum perforatum L. \\
\hline $\begin{array}{c}\text { Saquinavir } \\
\text { Chlorzoxazon }\end{array}$ & $\begin{array}{l}\text { Allium sativum L. } \\
\text { Allium sativum L. }\end{array}$ \\
\hline Felodipin & Mentha piperita L. \\
\hline Cyclosporine & Mentha piperita L. \\
\hline $\begin{array}{l}\text { Lithium } \\
\text { Carbamazepin }\end{array}$ & $\begin{array}{l}\text { Plantago sp. } \\
\text { Plantago sp. }\end{array}$ \\
\hline Alprazolam & Piper methysticum G. Forst. \\
\hline Benzodiazepine & Valeriana officinalis L. \\
\hline Echinacea & Echinaceae purpurea P.E. \\
\hline Sympaticomimetics & Ephedra sinica E.sp. \\
\hline Halothan & Ephedra sinica E.sp. \\
\hline Cyclopropane & Ephedra sinica E.sp. \\
\hline Heparine & Ginkgo biloba \\
\hline Acethylsalicylic acid & Ginkgo biloba \\
\hline Clopidogrel & Ginkgo biloba \\
\hline Warfarine & Panax ginseng \\
\hline Furosemid & Panax ginseng \\
\hline Phenelzin & Panax ginseng \\
\hline Spironolacton & Glycyrrhiza glabra \\
\hline Prednisolon & Glycyrrhiza glabra \\
\hline Hydrocortison & Glycyrrhiza glabra \\
\hline
\end{tabular}

resource for the future discovery of pharmaceutical agents.

It is difficult to discover new chemical entities from natural sources. However, it is also doubtful whether innovative compounds have been identified using combinatorial chemistry and HTS. In fact, many hits have been generated though none so far has reached advanced clinical development from a poor quality chemical library. However, there were several negative statements.

The success of natural products in drug discovery is essentially related to their complex chemical structure, to their existing in various species in nature, to the existing of high technology methods which are available, essen- 
tially for pharmaceutical companies and research centers.

Moreover, natural products have been, and will be, important sources of new pharmaceutical compounds. Recently, there has been a renewed interest in natural product research due to the failure of alternative drug discovery methods to deliver many lead compounds in key therapeutic areas, such as immunosupression, antiinfectives and metabolic diseases. It is clear that demand for drugs, disposable consumer products, biological agents and insecticides will continue to increase for the foreseeable future. But unless we take specific action to protect and develop our environment under sustainable conditions, the window of opportunity for the discovery of new medicinal and biological agents will be shut forever.

\section{REFERENCES}

[1] M. Lahlou, "Screening of Natural Products for Drug Discovery," Expert Opinion on Drug Discovery, Vol. 2, No. 5, 2007, pp. 697-705. doi:10.1517/17460441.2.5.697

[2] B. Patwardhan, A. D. B. Vaidya and M. Chorghade, "Ayurveda and Natural Products Drug Discovery,” Current Science, Vol. 86, No. 6, 2004, pp. 789-799.

[3] L. J. Nisbet and M. Moore, "Will Natural Products Remain an Important Source of Drug Research for the Future?” Current Opinion in Biotechnology, Vol. 8, No. 6, 1997, pp. 708-712. doi:10.1016/S0958-1669(97)80124-3

[4] E. J. Jacob, "Natural Products-Based Drug Discovery: Some Bottlenecks and Considerations," Current Science, Vol. 96, No. 6, 2009, pp. 753-754.

[5] M. S. Butler, "The Role of Natural Product Chemistry in Drug Discovery,” Journal of Natural Products, Vol. 67, No. 12, 2004, pp. 2141-2153. doi:10.1021/np040106y

[6] Y. W. Chin, M. J. Balunas, H. B. Chai and A. D. Kinghorn, "Drug Discovery from Natural Sources," The American Association of Pharmaceutical Scientists Journal, Vol. 8, No. 2, 2006, pp. 239-242.

[7] J. H. Shen, X. Y. Xu, F. Cheng, et al., "Virtual Screen- ing on Natural Products for Discovering Active Compounds and Target Information,” Current Medicinal Chemistry, Vol. 10, No. 21, 2003, pp. 2327-2342. doi:10.2174/0929867033456729

[8] C. Mahidol, S. Ruchirawat, H. Prawa, et al., "Biodiversity and Natural Product Drug Discovery," Pure and Applied Chemistry, Vol. 70, No. 11, 1998, pp. 2065-2072. doi:10.1351/pac199870112065

[9] M. K. Lutz, "Putting Nature Back into Drug Discovery," Nature Biotechnology, Vol. 21, No. 3, 2003, pp. 602-604. doi:10.1038/nbt0603-602

[10] L. J. Nisbet, M. Moore and D. D. Soejarto, "Will Natural Products Remain an Important Source of Drug Research for the Future?" Current Opinion in Biotechnology, Vol. 8, No. 6, 1997, pp. 708-712. doi:10.1016/S0958-1669(97)80124-3

[11] A. L. Harvey, "Medicines from Nature: Are Natural Products Still Relevant to Drug Discovery?” Trends in Pharmacological Sciences, Vol. 20, No. 5, 1999, pp. 196-198. doi:10.1016/S0165-6147(99)01346-2

[12] E. K Frank, G. T. Carter, "The Evolving Role of Natural Products in Drug Discovery,” Nature, Vol. 4, No. 3, 2005, pp. 206-220. doi:10.1038/nrd1657

[13] J. Littleton, “The Future of Plant Drug Discovery,” Expert Opinion on Drug Discovery, Vol. 2, No. 5, 2007, pp. 673-683. doi:10.1517/17460441.2.5.673

[14] G. R. Dietzman, "Information Handling for Natural Products Acquisitions," Screening Forum, Vol. 3, No. 4, 1995, p. 11.

[15] G. L. Verdine, “The Combinatorial Chemistry of Nature,” Nature, Vol. 384, Supplement 6604, 1996, pp. 11-13.

[16] M. Lahlou, "De la Plante au Médicament: Une Passerelle entre Tradition et Science,” Édition LAWNE, Maroc. ISBN 978-9954-31-103-5, 2012, p. 272.

[17] D. S. Fabrican, N. R. Farnsworth, “The Value of Plants Used in Traditional Medecine for Drug Discovery," Environmental Health Perspectives, Vol. 109, Supplement 1, 2001, pp. 69-75. 